\title{
Erratum to: Nonclassical properties of two families of $q$-coherent states in the Fock representation space of $q$-oscillator algebra
}

\section{H. Fakhri ${ }^{\mathrm{a}}$ (D), S. E. Mousavi-Gharalari ${ }^{\mathrm{b}}$}

Department of Theoretical Physics and Astrophysics, Faculty of Physics, University of Tabriz, P. O. Box 51666-16471, Tabriz, Iran

Received: 3 May 2020 / Published online: 18 June 2020

(C) Società Italiana di Fisica and Springer-Verlag GmbH Germany, part of Springer Nature 2020

\section{Erratum to: Eur. Phys. J. Plus (2020) 135:253 https://doi.org/10.1140/epjp/s13360-020-00265-3}

Unfortunately, after publication, we found some misprints in Eur. Phys. J. Plus (2020) 135: 253. We list them here because their number is not few.

After publication of paper [1], in which signal-to-quantum noise ratios, Mandel parameters and the second-order correlation functions have been investigated, the authors realized that some relations in Eqs. (5a), (5b), (14b), (14c) and (16a)-(16d) needed to be corrected. Their corrected versions are as the following, respectively:

$$
\begin{gathered}
\left(q^{\frac{N}{2}} a\right)|z\rangle_{I}=\frac{z}{\sqrt{1+(1-q)|z|^{2}}}\left|q^{\frac{1}{2}} z\right\rangle_{I}, \\
a|z\rangle_{I I}=\frac{z}{\sqrt{1+\left(1-q^{-1}\right)|z|^{2}}}\left|q^{\frac{-1}{2}} z\right\rangle_{I I} \cdot \\
\sigma_{|z\rangle_{I}}^{\left(X_{I I}\right)}=\frac{\frac{(2 \operatorname{Real}(z))^{2}}{1+(1-q)|z|^{2}} \frac{\left(E_{q}\left((1-q) q^{\frac{1}{2}}|z|^{2}\right)\right)^{2}}{E_{q}\left((1-q)|z|^{2}\right) E_{q}\left((1-q) q|z|^{2}\right)}}{1+\frac{(1+q)|z|^{2}+2 q^{\frac{1}{2}} \operatorname{Real}\left(z^{2}\right)}{1+(1-q)|z|^{2}}-\frac{(2 \operatorname{Real}(z))^{2}}{1+(1-q)|z|^{2}} \frac{\left(E_{q}\left((1-q) q^{\frac{1}{2}}|z|^{2}\right)\right)^{2}}{\left.E_{q}(1-q)|z|^{2}\right) E_{q}\left((1-q) q|z|^{2}\right)}} \\
|z|<\infty \\
\sigma_{|z\rangle_{I I}}^{\left(X_{I}\right)}=\frac{(2 \operatorname{Real}(z))^{2}}{1+\left(1-q^{-1}\right)|z|^{2}} \frac{\left(e_{q}\left((1-q) q^{-\frac{1}{2}}|z|^{2}\right)\right)^{2}}{e_{q}\left((1-q)|z|^{2}\right) e_{q}\left((1-q) q^{-1}|z|^{2}\right)} \\
1+\frac{\left(1+q^{-1}\right)|z|^{2}+2 q^{-\frac{1}{2}} \operatorname{Real}\left(z^{2}\right)}{1+\left(1-q^{-1}\right)|z|^{2}}-\frac{(2 \operatorname{Real}(z))^{2}}{1+\left(1-q^{-1}\right)|z|^{2}} \frac{\left(e_{q}\left((1-q) q^{-\frac{1}{2}}|z|^{2}\right)\right)^{2}}{e_{q}\left((1-q)|z|^{2}\right) e_{q}\left((1-q) q^{-1}|z|^{2}\right)}
\end{gathered}
$$

The original article can be found online at https://doi.org/10.1140/epjp/s13360-020-00265-3.

\footnotetext{
a e-mail: hfakhri@tabrizu.ac.ir (corresponding author)

b e-mail: e.mousavi@tabrizu.ac.ir
} 


$$
\begin{array}{ll}
|z|<\frac{1}{\sqrt{1-q}} . & \\
Q_{\left.|z\rangle_{I}^{\left(q^{\frac{N}{2}}\right.} a\right)}=\frac{-q|z|^{5}(q-1)^{2}+|z|^{3}\left(q^{2}-1\right)}{q|z|^{4}(q-1)^{2}-|z|^{2}\left(q^{2}-1\right)+1}, & |z|<\infty . \\
Q_{|z\rangle_{I I}^{(a)}}^{(a)}=\frac{-|z|^{4}(q-1)^{2}-|z|^{2} q\left(q^{2}-1\right)}{|z|^{4}(q-1)^{2}+q|z|^{2}\left(q^{2}-1\right)+q^{3}}, & |z|<\frac{q}{\sqrt{1-q}}, \\
g_{|z\rangle_{I}{ }^{(2)\left({ }^{\frac{N}{2}} a\right)}(0)=\frac{q|z|^{2}(q-1)-q}{q|z|^{2}(q-1)-1},} & |z|<\infty, \\
g_{|z\rangle}^{(2)(a)}(0)=\frac{|z|^{2}(q-1)+q}{|z|^{2}(q-1)+q^{2}}, & |z|<\frac{q}{\sqrt{1-q}} .
\end{array}
$$

Since the discussions after Eqs. (14) and (16) were done with the correct forms, the results in the original paper are correct. It is necessary to mention that Fig. 2a-d has been plotted for $\theta=\pi$ in the original paper. Furthermore, we must clarify that the $q$-coherent states $|z\rangle_{I I}$ exhibit the super-Poissonian statistics and bunching effect via operator $a$ in $|z|<\frac{q}{\sqrt{1-q}}$. In Fig. 2b, the correct range for the $\sigma_{|z\rangle_{I}}^{\left(X_{I I}\right)}$ is from 0 to 6 .

\section{Reference}

1. H. Fakhri, S.E. Mousavi-Gharalari, Nonclassical properties of two families of $q$-coherent states in the Fock representation space of $q$-oscillator algebra. Eur. Phys. J. Plus 135, 253 (2020). https://doi.org/10.1140/ epjp/s13360-020-00265-3 